\title{
UV AND OPTICAL SPECTROSCOPIC OBSERVATIONS OF COLLIDING WINDS IN WR+O BINARIES
}

\author{
NICOLE ST-LOUIS \\ Département de Physique, Université de Montréal \\ C.P. 6128, Succ. Centre Ville, Montréal (Québec), H3C 3J7, Canada \\ and Observatoire du Mont Mégantic
}

\begin{abstract}
In this paper, a review of spectroscopic evidence for colliding wind effects in $\mathrm{WR}+\mathrm{O}$ binaries is presented with a particular emphasis on variability at ultraviolet wavelengths. In the optical, evidence for colliding winds comes from extra emission formed in the region of shocked gas and superimposed on the strong emission lines formed in the WR wind. At ultraviolet wavelengths, we observe the consequences of the fact that between the two stars the winds are prevented from reaching their terminal velocity because of the wind-wind interaction.
\end{abstract}

Key words: stars: Wolf-Rayet - binaries - colliding winds

\section{Introduction}

When studying hot stars in close binary systems, interaction effects between the winds of the two stars can hardly be disregarded. In particular, as a consequence of the collision between the two dense outflows, a shock region is formed which is coned-shaped and surrounds the component which has the wind with the smaller momentum flow. Although this has been recognized for some time, only very little observational work has been done to try to detect the consequences of the presence of such a structure.

The most widely known observational signature of the collision between two hot-star winds is the detection of high-energy photons. The soft X-ray flux from the shock-heated gas in the wind interaction region was found to be much smaller than predicted by models. This has prompted improvements to the theory; Stevens \& Pollock (these proceedings) have shown that, for V444 Cygni (WN5+06), the predicted levels of X-rays are more compatible with the observed values if one takes into account the radiation field from the O-star companion on the WR-star wind.

In the past ten years, efforts have been made to observe colliding winds through spectroscopic phase-dependent variations of $\mathrm{WR}+\mathrm{O}$ binaries, both at optical and ultraviolet wavelengths. Table 1 lists the ten binaries for which evidence for colliding winds has been found. For each system, the WR number, the spectral type and orbital period are given. The last column indicates if the variations were found at optical and/or ultraviolet wavelengths.

In this paper, a brief description of the variability for the various binaries listed in table 1 will be presented. Most of the phase-dependent spectroscopic 
TABLE I

Spectroscopic evidence for colliding winds in $\mathrm{WR}+\mathrm{O}$ binaries.

\begin{tabular}{cccccc}
\hline WR number & other name & spectral type & period (d) & reference & UV/opt \\
\hline 11 & $\gamma$ Velorum & WC8+O9I & 78.5002 & 1,9 & UV \& opt \\
21 & HD 90657 & WN4+O4-6 & 8.255 & 2 & UV \\
22 & HD 92740 & WN7+a & 80.35 & 9 & UV \\
79 & HD 152270 & WC7+O5-8 & 8.893 & 3 & opt \\
133 & HD 190918 & WN4.5+O9.5Ib & 112.8 & 4 & opt \\
139 & V444 Cygni & WN5+O6 & 4.21238 & $2,5,6,8$ & UV \& opt \\
140 & HD 193793 & WC7+O4-5V & 2900 & 10,11 & UV \& opt \\
151 & CX Cep & WN5+O5V & 2.1269 & 7 & opt \\
153 & GP Cep & WN6+O & 6.6884 & 2 & UV \\
155 & CQ Cep & WN7+a & 1.64 & 8 & UV \& opt \\
& Br 22 (LMC) & WC6:+O & 14.926 & 12 & opt \\
\hline
\end{tabular}

References: 1. St-Louis, Willis \& Stevens 1993; 2. Koenigsberger \& Auer 1985; 3. Lührs (1991); 4. Bertrand (1994); 5. Shore \& Brown (1988); 6. Marchenko, Moffat \& Koenigsberger 1994; 7 . Lewis et al. 1993; 8. Marchenko, these proceedings; 9 . this paper; 10. Williams et al. 1987; 11. Hervieux, these proceedings; 12 . Bartzakos, these proceedings.

variations observed in the optical and attributed to colliding winds are excess emission from the region of shocked gas. In this paper, the discussion will concentrate on variations found at ultraviolet wavelengths as most of the evidence for colliding winds at optical wavelengths is discussed elsewhere in these proceedings.

\section{WR21 and WR153}

Spectroscopic evidence for colliding winds in WR21 and WR153 comes from the work of Koenigsberger (1983) and Koenigsberger \& Auer (1985). These authors found phase-dependent variations for five $\mathrm{WR}+\mathrm{O}$ binaries using low resolution IUE spectra. The observed changes mainly consist in a strengthening of the absorption components of the major P-Cygni profiles at phases when the $\mathbf{O}$ star is behind the WR star. These variations were interpreted as being the result of selective wind eclipses of the 0 -star continuum by atoms in the WR-star wind.

Concurrently with the increased strength of these P-Cygni absorptions the authors also found that the $\mathrm{C}$ IV $\lambda 1550$ absorption component becomes narrower for three systems, WR21, WR139 and WR153 when the 0 star companion is behind the WR star. These changes were interpreted has being a consequence of the collision of the two stellar winds. In a WR+O binary in which the $O$ star has a significant wind, the observed C IV $\lambda 1550$ P-Cygni 
profile is the combination of the contributions from the $\mathrm{O}$ - and WR-star winds. If the star with the highest terminal velocity has a saturated C IV $\lambda 1550$ absorption component, the absorption component from the other star cannot be distinguished. The only variations that should be observed as a function of phase are those caused by selective wind eclipses.

However, it is possible that for close binaries the winds are prevented from reaching their terminal velocities in the region between the two stars because of the collision of the two stellar outflows. When the star with the faster wind (in this case the $\mathrm{O}$ star) is in front of it's companion, we see in absorption the unattenuated wind. When it is behind, we see in absorption the region between the two stars and as the terminal velocity is reduced, the blue wing of the absorption component moves to a smaller velocity (or a longer wavelength) resulting in a narrower profile than at phases when the $\mathrm{O}$ star is in front. The result is a narrowing of the $\mathrm{C}$ IV P-Cygni absorption component at phases when the $\mathrm{O}$ star is behind the WR star, as is observed for WR21, WR139 and WR153. A simultaneous strengthening of the absorption component should theoretically be observed but the CIV profiles are saturated for these three stars.

\section{WR22}

WR22 is an 80-day binary system in which very little is known about the O-type companion. A new orbit has recently been obtained by Rauw et al. (these proceedings) and is adopted here. Evidence for colliding winds is scanty and therefore this system is a good candidate to include in future spectroscopic colliding-wind studies. Figure 1 presents the Si IV $\lambda 1402$ profile from the IUE high-resolution spectra of WR22 available in the archives. The thick line is a mean of seven observations obtained in December 1985 ( $\phi=$ 0.80 ) and for which no variability was found. Each of the other four spectra is represented with a thin line and compared with this mean. Successive observations were shifted in ordinate for clarity.

The evidence for colliding winds in this system comes from the variation of the blue-edge velocity of the Si IV absorption component as a function of phase. When the WR star is in back of the $O$ star near phase 0.5 , we see a slower wind because between the two stars the outflow is prevented from reaching its terminal velocity. At phase 0.80 on the other hand the portion of the WR wind that we see in absorption is unattenuated and we can then see the full extent of the wind.

\section{The eclipsing binary V444 Cygni}

V444 Cygni is one of the best studied WR+O binaries. The fact that it is an eclipsing system has allowed for a very good determination of the orbit 


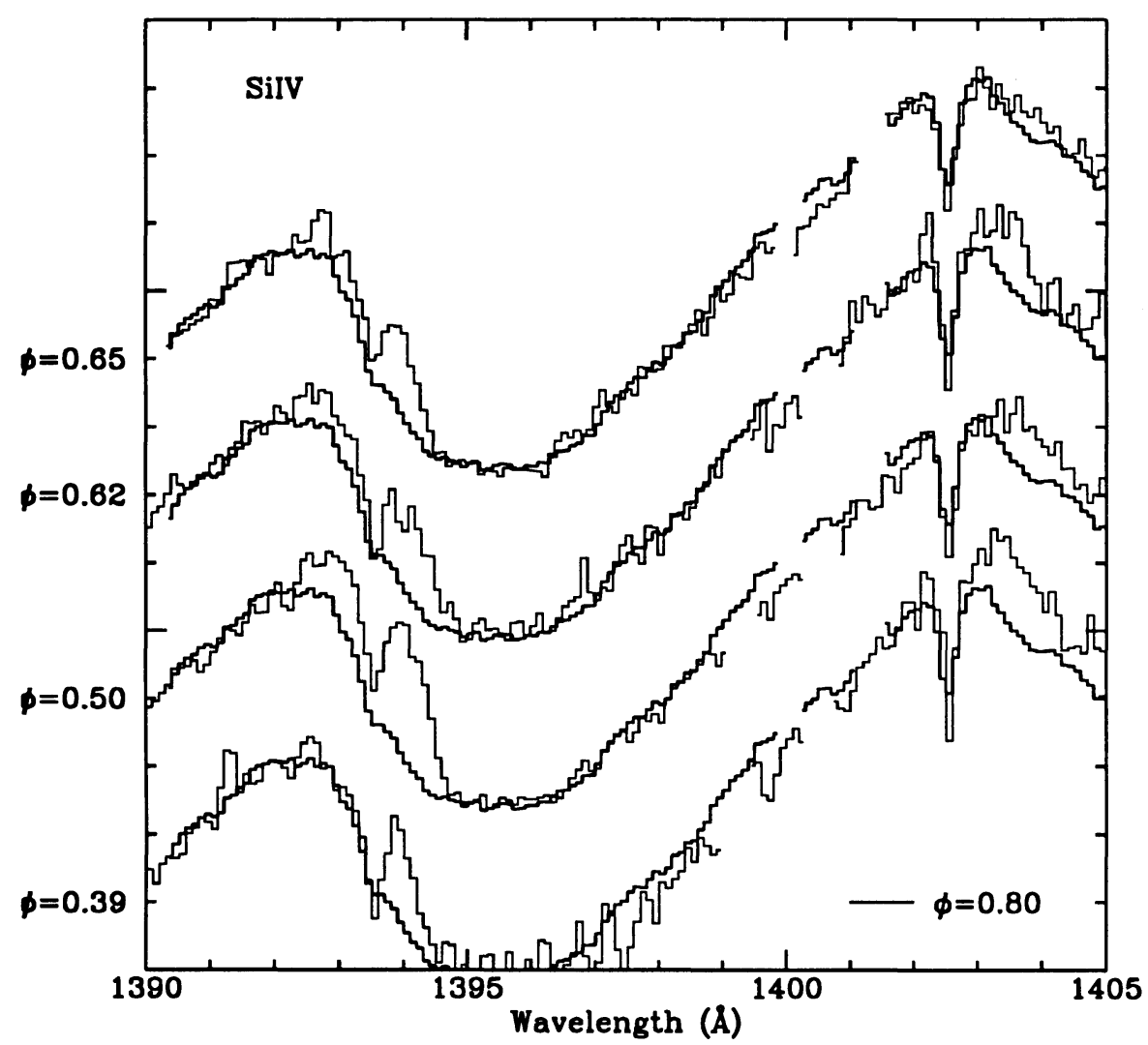

Fig. 1. IUE high-resolution spectra of WR22. The thick line is the mean of 7 observations at phase 0.8 and each thin line is a spectrum obtained at the orbital phase indicated on the left of the graph. Each successive spectrum was shifted in ordinate for clarity.

and for a good handle on the stellar parameters. Koenigsberger (1983) and Koenigsberger \& Auer (1985) were the first to present spectroscopic evidence for colliding winds based on low-resolution IUE spectra of the system. However a more detailed study was presented by Shore and Brown (1988) based on a series of high-resolution IUE spectra.

Shore \& Brown (1988) found that near secondary eclipse, when the $O$ star is in front of the WR star, the P-Cygni absorption components of the N IV $\lambda 1718$ and He II $\lambda 1640$ P-Cygni profiles disappear and a high-velocity wing appears on the blue edge of the P-Cygni absorption component of $\mathrm{C}$ IV $\lambda 1550$. These changes are compatible with those found by Koenigsberger \& Auer (1985) at lower resolution and which they interpreted as being caused by a combination of selective wind eclipse and the presence of colliding winds which prevented the WR and $O$ star outflow to reach their terminal velocity 
in the region between the two stars. Shore \& Brown (1988), however, do not invoke selective wind eclipses. Instead they explain all the observed changes by the presence of a lower-density coned-shaped cavity in the WR wind created by the shock surface formed as a consequence of the wind-wind collision. The disappearance of the absorption components is attributed to the fact that when this cavity is in front of the WR star, the lower density of the gas does not allow for the development of a significant absorption component for the He II and N IV transitions. At those same phases we see high-velocity material moving along the shock surface in projection on the $\mathrm{O}$-star disk. This produces a high velocity wing on the blue edge of the $\mathrm{C}$ IV $\lambda 1550$ P-Cygni profile.

The authors therefore predict that the appearance of this high-velocity wing should be very sudden and that it should last for only a very short phase interval since the shock surface is predicted to be relatively narrow. New observations with a much better phase coverage are required to discriminate between this interpretation and the one of Koenigsberger \& Auer (1985) in which a more gradual and long-lasting appearance of the high-velocity wing is expected.

\section{5. $\quad$ WR11 $=\gamma$ Velorum}

$\gamma$ Velorum is a well-known binary system composed of a WC8 star and a 09 supergiant. In spite of a relatively long orbital period $(78.5 \mathrm{~d})$ and a surprisingly low soft X-ray flux, spectroscopic evidence for colliding winds has been discovered by St-Louis, Willis \& Stevens (1993). Phase-dependent variations were detected in a series of IUE high-resolution archival spectra. For most of the resonance and low-excitation lines found in the UV spectrum of $\gamma$ Velorum, these authors observe a gradual strengthening of the P-Cygni absorption components and/or weakening of emission lines as the $\mathrm{O}$-star companion orbits to the back of the WR wind. At the same time, the absorption component of P-Cygni profiles such as C IV $\lambda 1550$ and Si IV $\lambda 1396$ become narrower.

The changes are interpreted as being caused by a combination of selective wind eclipses and the presence of a coned-shaped cavity produced by the collision between the two stellar winds. The narrowing of the absorption components of resonance lines constitute once again, the evidence for colliding winds. In this case, the appearance of the high-velocity wing is gradual and therefore is not compatible with material moving along the coned-shaped shock surface. Instead, the authors favor the interpretation of Koenigsberger \& Auer (1985) in which the winds of the two stars are prevented from reaching their terminal velocity in the region between the two stars. Moreover, as the separation between the two star is always quite large $\left(>200 \mathrm{R}_{\odot}\right)$ in this eccentric system, the disappearance of the absorption 
components and the weakening of the emission lines when the 0 star is in front cannot be explained by the presence of a lower-density cavity in the WR wind as the line formation regions are mostly situated closer to the star.

We have obtained a new IUE dataset of high-resolution spectra of $\gamma$ Velorum at selected orbital phases. These observations mainly confirm the results from the previous dataset. In addition to the gradual increase of the blueedge velocity of the P-Cygni absorption components of resonance lines such as $\mathrm{C}$ IV $\lambda 1550$ as the $\mathrm{O}$ star orbits to the front of the WR star, the gradual strengthening of the absorption components or weakening of emission lines for other transitions occurs over a gradually larger wavelength interval as the $O$ star orbits to the back of the WR wind. This is exactly what is expected for selective wind eclipses.

We have also obtained an optical dataset of which only a small subset is presented here. The spectra were obtained using the $61 \mathrm{~cm}$ Helen Sawyer Hogg telescope of the University of Toronto in Las Campanas, Chile. The complete set of observations will be analyzed elsewhere (St-Louis, in preparation). Ten spectra of $\gamma$ Velorum obtained in January 1993 and covering a wavelength range from $5200 \AA$ to $6200 \AA$ are presented in Figure 2. The orbital phases at which the observations were obtained are indicated on the left of the graph and are distributed over the 0.13 to 0.35 interval. Each spectrum is overploted on the first in the series in order to facilitate the comparison. A gradual increase in the total flux of $\mathrm{C}$ III $\lambda 5696$ and $\mathrm{He} I$ $\lambda 5876$ is clearly visible from phase 0.13 to phase 0.35 , i.e., when the 0 star orbits to the front of the WR star. This is similar to what was found in the ultraviolet and interpreted as being due to selective wind eclipses of the $\mathrm{O}$-star continuum by the WR-star wind. In comparison, the higher excitation line of $\mathrm{C}$ IV $\lambda 5807$ does not vary significantly as was also found for similar transitions in the ultraviolet. This is explained by significantly lower densities; these higher excitation transitions are mostly formed closer to the star and the inclination of the system is too low to allow an eclipse of this material. However, the amplitude of variation is much smaller for optical lines than for ultraviolet transitions. While the equivalent width of the $\mathrm{C}$ III $\lambda 5696$ line changed by $10 \%$ over the $0.13-0.35$ phase interval, that of $\mathrm{C}_{\text {III }}$ $\lambda 2297$ changed by a factor of three.

Variations in the shape of the $\mathrm{CIII}$ and He I profiles are also visible which could be compatible with extra emission from the region of shocked gas viewed at different velocities as a function of orbital phase. However these phase-dependent profile changes need to be checked for repeatability and details of the nature of the variability as a function of phase should be confronted with model predictions. 


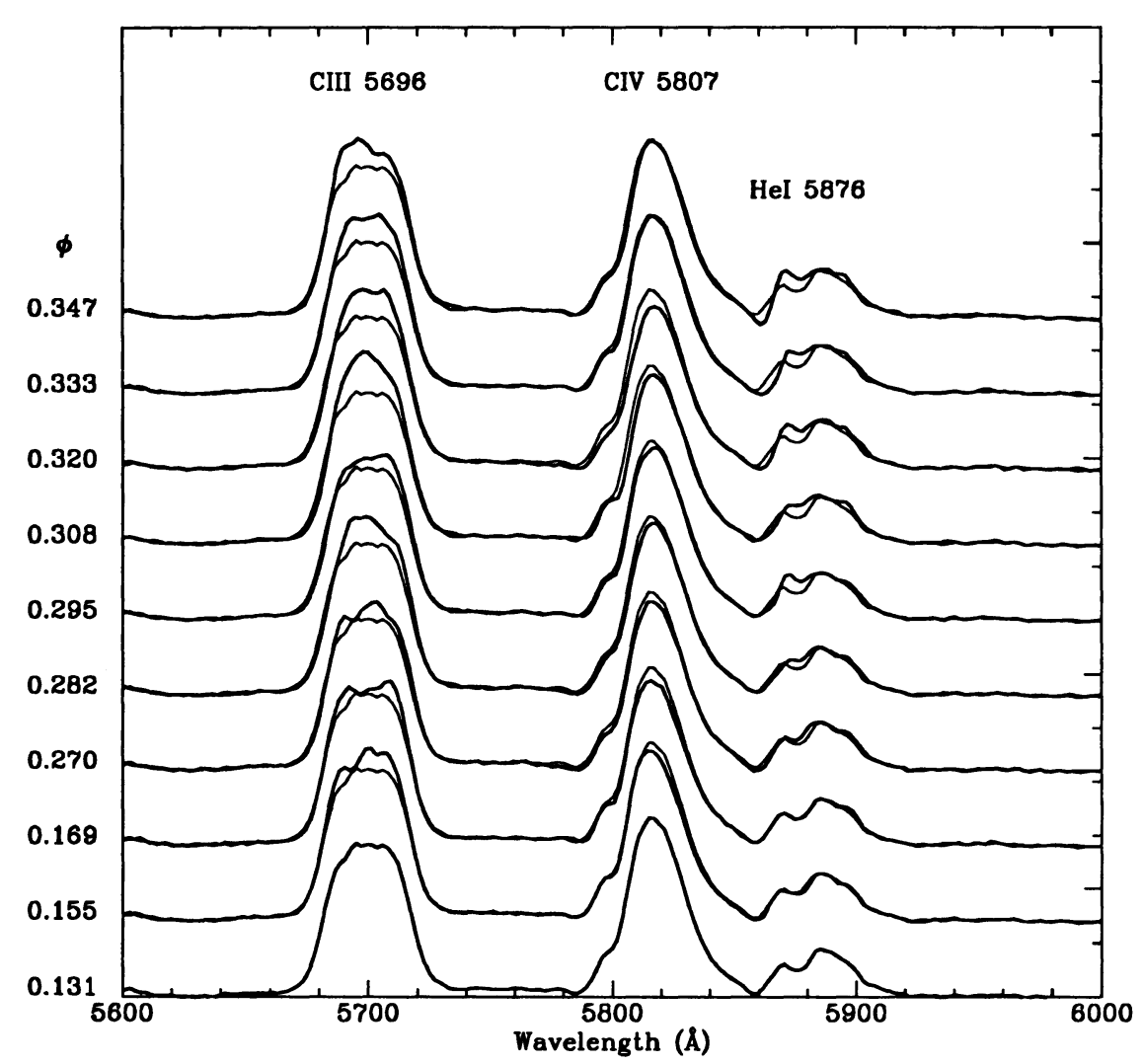

Fig. 2. Optical spectra of $\gamma$ Velorum as a function of orbital phase.

\section{Conclusions}

Spectroscopic evidence for colliding winds has been found for $10 \mathrm{WR}+\mathrm{O}$ binary systems at optical and ultraviolet wavelengths.

In the optical, phase-dependent variations in the shape of various emission lines are observed. The changes are caused by extra emission superimposed on the strong emission lines from the WR wind. The emission is produced from shock-heated gas and is viewed at different velocities as a function of orbital phase. For WN systems, the changes are found in various lines such as He II $\lambda 4686$ and many He I lines. For WC systems, most of the variations have been found in the $\mathrm{C}_{\text {III }} \lambda 5696$ line (and He I $\lambda 5876$ line for $\gamma$ Velorum).

In the ultraviolet, evidence for colliding stellar winds comes from a gradual increase of the velocity of the blue wing of the P-Cygni absorption components of resonance transitions such as CIV $\lambda 1550$ and Si IV $\lambda 1396$. The changes are thought to be due to the fact that, in the region between the 
two stars, the winds are prevented from reaching their terminal velocities because of the presence of a cone-shaped interaction region formed as a consequence of the wind-wind collision.

\section{References}

Bertrand, J.-F. 1994, MSc thesis, Université de Montréal

Lührs, S. 1991, PhD thesis, University of Münster

Koenigsberger, G. 1983, PhD thesis, Pennsylvania State University

Koenigsberger, G., Auer, L.H. 1985, $A p J$ 297, 255

Lewis, D., Moffat, A.F.J., Matthews, J.M., Robert, C., Marchenko S.V. 1993, ApJ 405, 312

Marchenko, S.V., Moffat, A.F.J., Koenigsberger, G. 1994, ApJ 422, 810

St-Louis, N., Willis, A.J., Stevens, I.R. 1993, $A p J$ 415, 298

Shore, S., Brown, D.N. 1988, ApJ 334, 1021

Williams, P.M., van der Hucht, K.A., van der Woerd, H., Wamsteker, W.M., Geballe, T.R., Garmany, C.D., Pollock, A.M.T. 1987, in: H.J.G.L.M. Lamers \& C.W.H. de Loore (eds.), Instabilities in Luminous Early Type Stars (Dordrecht: Reidel), p. 221

\section{DISCUSSION:}

Moffat: From your polarization studies (St-Louis et al. 1987, 1988), updated by more recent work (see my talk), $\gamma$ Vel was one system in which $\dot{M}(\mathrm{WR})$ could be almost a full order of magnitude lower than the radio $\dot{M}(W R)$ you used to interpret the wind collisions vs. wind eclipses on this system. What would the impact be on your interpretation of $\gamma$ Vel if $\dot{M}$ (WR) were decreased by a factor $\sim 10$ ?

St-Louis: If those lower mass-loss rates are confirmed, it could help to lift the shock off the surface of the $O$ star and perhaps help to explain why we see the terminal velocity of the $O$ star at phases 0.3 and 0.6 .

Owocki: You mentioned the effect described by Stevens \& Pollock in a poster here that the wind flow between binary $\mathrm{O}$-stars may be slowed substantially by the radiative force from the opposite star, thus reducing the strength at the collision shock, and so helping explain the low flux and softness of observed X-rays. In WR + $\mathrm{O}$ systems, this effect might provide a way to learn what makes the mass loss from WR stars a factor ten higher for a given luminosity. Namely, this WR wind enhancement might be due either to (1) a difference in the WR flux spectrum or (2) a ten-times larger WR wind line opacity (arising somehow from the low $\mathrm{H}$ abundance). Now consider a WR $+\mathrm{O}$ binary with each star having similar luminosities, but with $\dot{\mathrm{M}}_{\mathrm{WR}} \approx 10 \dot{\mathrm{M}}_{\mathrm{o}}$. In case 1, the O-star should have little effect in slowing down the WR wind, which should then give a bow shock that is tightly wrapped onto the O-star. However, in case 2 the $\mathrm{O}$-star radiation should be able to substantially decelerate the WR wind, and thus cause the bow-shock to be both weakened, and shifted to closer to the WR $+\mathrm{O}$ mid-point. I would think that careful interpretation of observed phase vatiations at UV line profiles might be able to distinguish between these two scenarios, and thus constrain the mechanism for driving the large WR wind mass loss. 
Cassinelli: The WR $+O$ binary systems are used to calculate the $\beta$ for the velocity law. However your picture with interaction cones seems quite complicated. Given that, can one really get a reliable estimate of $\beta$ ?

St-Louis: Of course the situation is much more complicated when one includes colliding winds. However, I think the effects of the shock cone and those of the selective atmospheric eclipses are relatively well understood. Therefore I think there is some hope of distinguishing the two and perhaps obtain $\beta$ from studies of the selective atmospheric eclipses taking into account the presence of the shock cone.

Koenigsberger: From the synthetic profile variations we have calculated for V444 Cyg (Auer and Koenigsberger, 1994, ApJ in press) the effects due to the wind-wind collision are a second order effect, compared with the effects due to wind eclipse.

Schmutz: I noticed on your plot of the ratio between phase 0 and 0.5 of $\gamma$ Vel that there is a feature due to CIII 1909. Given the low oscillator strength of this line, are you still able to explain this feature as an eclipse effect?

St-Louis: The variability is explained by absorption through the wind so even though the oscillator strength is very low, the density of CIII is so high that it's easy to explain the variations.

Willis: The atmosphere eclipse effect in $\gamma$ Vel can be seen in the intercombination line CIII] 1909, despite its low f-value, because (i) the WC8 mass loss rate is high, leading to high CIII column-density, (ii) the high abundance of carbon in the WC8 wind. Modelling the eclipse effect observed in CIII] 1909 in $\gamma$ Vel gives a WC8 mass loss rate of about $7 \times 10^{-5} \mathrm{M}_{\circ} \mathrm{y}^{-1}$, consistent with $\mathrm{M}$ from the radio, and this confirms that eclipses in the intercombination line is observed, as first pointed out by Willis \& Wilson $(1976$, A\&A, 47, 429) who first identified this phenomenon of selective wind line eclipses in WR+O systems.

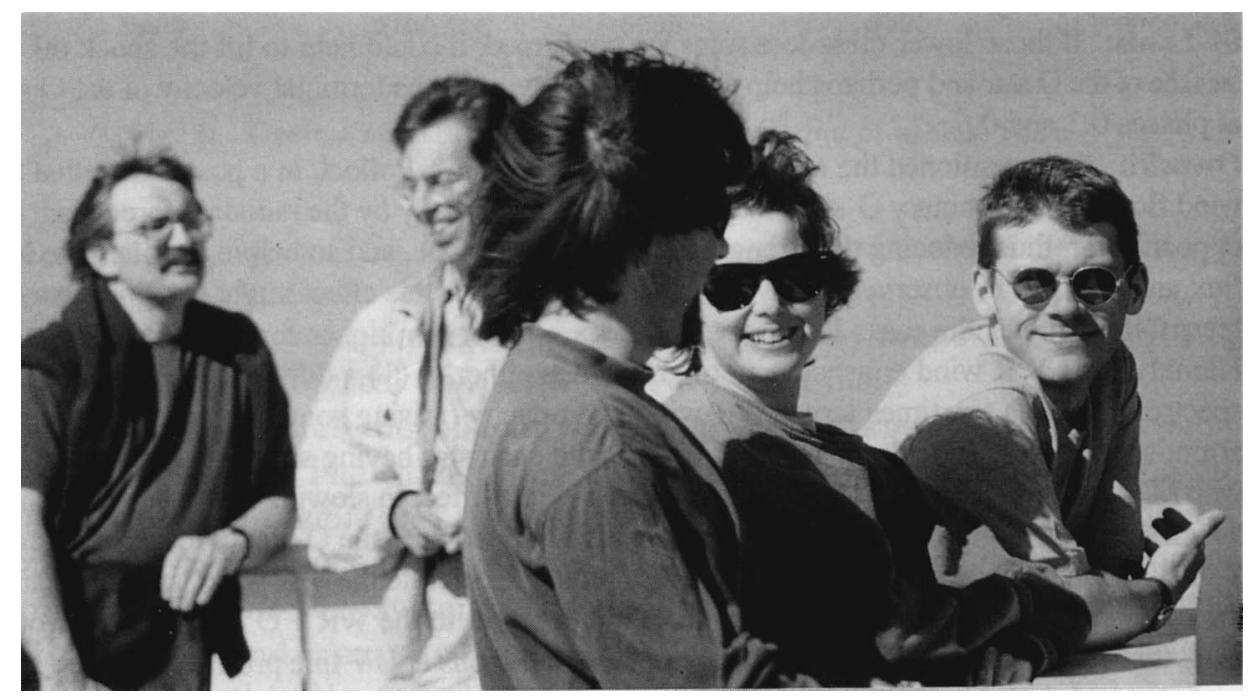

Pollock, St-Louis, Crowther 